\title{
MiR-1b up-regulation inhibits rat neuron proliferation and regeneration yet promotes apoptosis via targeting KLF7
}

\author{
Xiaojie $\mathrm{Li}^{1}$, Lihe Yuan ${ }^{1}$, Jin Wang ${ }^{1}$, Zun Zhang ${ }^{2}$, Shaojing $\mathrm{Fu}^{1}$, Shaobin Wang ${ }^{1}$, Xinhui Li ${ }^{3}$ \\ ${ }^{1}$ Department of Neurology, Inner Mongolia Baogang Hospital, China, ${ }^{2}$ Department of Orthopaedics, Inner Mongolia Baogang Hospital, \\ China, ${ }^{3}$ Department of Neurology, The First Affiliated Hospital of Baotou Medical College, China
}

\begin{abstract}
Introduction: MicroRNA (miRNA) is known to be involved in nerve injury. Our study aimed to identify the role and mechanism of miR-1b in rat neuron proliferation, regeneration and apoptosis.

Material and methods: Neurons were successfully separated and identified using a microscope and immunofluorescence staining of microtubule-associated protein 2 (MAP-2). The expressions of miR-1b and Krüppel-like factor 7 (KLF7) were detected by quantitative real-time polymerase chain reaction (qRT-PCR). Neuron viability and apoptosis were detected by MTT assay and flow cytometry, respectively. Neuron regeneration states were observed using a microscope and analysed by the Imagel software. Expressions of C-caspase-3 and cell regeneration-related proteins (nerve growth factor [NGF], ciliary neurotrophic factor [CNTF] and brain-derived neurotrophic factor [BDNF]) were measured by Western blot. Target genes and potential binding sites of KLF7 and miR-1b were predicted by TargetScan 7.2 and confirmed by dual luciferase reporter assay.

Results: Neurons were identified as MAP-2-positive. Up-regulation of miR-1b reduced neuron viability and regenerative ability, promoted neuron apoptosis and C-caspase-3 expression, and down-regulated the expressions of cell regeneration-related proteins. KLF7 was the target gene of miR-1b. Overexpressed KLF7 rescued the effects of up-regulation of miR-1b on neuron viability, regeneration and apoptosis. Expressions of NGF, CNTF and BDNF were suppressed yet $C$-caspase-3 expression was up-regulated by miR-1b mimic, which was partially rescued by overexpressed KLF7.
\end{abstract}

Conclusions: Up-regulation of miR-1b promoted rat neuron proliferation and regeneration yet inhibited apoptosis via targeting KLF7.

Key words: miR-1b, Krüppel-like factor 7, neuron, cell regeneration.

\section{Introduction}

The main function of neurons is to process and transmit information, which is realized by integrating information across thousands of their synaptic inputs [1]. A large number of neuron deaths take place during development and pathology, especially due to the limited ability of adult neurons to proliferate or be replaced [7]. Currently, although considerable progress has been obtained in curing neuron apoptosis and regeneration, many questions about their mechanisms remain to be answered [23].

Communicating author:

Xinhui Li, Department of Neurology, The First Affiliated Hospital of Baotou Medical College, No. 41, Linmeng Road, Kundulun District, Baotou City, Inner Mongolia Autonomous Region, 014010, China, phone: 86-0472-2178486, e-mail: lixinhui_xinhuil@163.com 
MicroRNAs (miRNAs) are a class of small non-protein coding RNA with 21-25 nucleotides in length, and function as essential regulators of gene expression by inhibiting the degradation of their target mRNAs and repressing translation [24]. MiRNAs that are located in local processes of neurons regulate protein synthesis, and their expression is regulated by synaptic activity [9]. Increasing evidence has shown that miRNAs are involved in neuron apoptosis and regeneration. For example, Chen et al. [5] found that rat neuron apoptosis was reduced by up-regulation of miR-23a after hypoxia exposure, suggesting that miR-23a may be a potential target for alleviating neuron apoptosis. In addition, it has been reported that miR-135s promoted central nervous system axon regeneration after nerve injury in adult mice via silencing Krüppel-like factor 4 (KLF4) [30]. Furthermore, some miRNAs have been reported to play a positive role in neuron apoptosis and regeneration. For instance, Gaudet et al. [8] discovered that miR-155 up-regulation decreased spontaneous axon growth from neurons after spinal cord injury. And it has been identified that down-regulated miR-1b promoted neuron regeneration of peripheral nerve injury by targeting NDRG3 [20]. Nevertheless, the function of miR-1b in neurons is still poorly studied. Therefore, our study focused on further unveiling the role of miR-1b in neuron apoptosis and regeneration.

Krüppel-like factor 7 (KLF7) is a member of the KLF family of zinc finger transcription factors, and has a low expression level in numerous human tissues [6]. Previous studies have shown that KLF7 regulated the lineages of the neuroectoderm and mesoderm and powerfully regulated the development of many other organs [4]. Moreover, Li et al. [18] found that KLF7 overexpression in bone marrow stromal stem cells graft transplantation promoted sciatic nerve regeneration, suggesting that KLF7 functioned as a positive role in curing neural injury. In addition, a previous study has revealed that inhibition of miR-146b promotes sciatic nerve regeneration via targeting KLF7 [19]. Therefore, as has been previously reported, miRNA-targeting techniques could be used as an alternative therapeutic approach to the exogenous application of KLF7 on neuron regeneration [33]. However, there are only limited studies on the relationship between miR-1b and its potential target KLF7 in neurons.

Taken together, in this study we set out to explore the effect of miR-1b on neurons and discover whether KLF7 is directly targeted by miR-1b and whether down-regulation of miR-1b affects neuron proliferation, regeneration and apoptosis.

\section{Material and methods \\ Ethics statement}

The guidelines of the China Council on Animal Care and Use were strictly followed during animal experiments. This study was approved by the Committee of Experimental Animals of Inner Mongolia Baogang Hospital (approval number IMBH20191106). The animal experiments were carried out in the Inner Mongolia Baogang Hospital. Maximum effort was made to minimize pain to the animals.

\section{Neuron isolation and culture}

Male Wistar rats (180-220 g, 18 days) were purchased from the Experimental Animal Centre of the Zhejiang Province. Firstly, the rats were soaked in $75 \%$ alcohol for about 3 minutes and then anesthetized with phenobarbital $(45 \mathrm{mg} / \mathrm{kg})$. Then the brain of the rats was taken out and placed in $8 \mathrm{ml}$ of phosphate-buffered saline (PBS) with high-glucose on ice, and the meninges and large blood vessels in the brain were removed. Subsequently, the rest of the brain was removed except for the cerebral cortex. The cerebral cortex was cut into small pieces of about 1 cubic millimetre, and then $500 \mu \mathrm{l}$ of $2.5 \%$ trypsin and $300 \mu \mathrm{l}$ of DNA enzyme was added. The cells were digested at $37^{\circ}$ with $5 \% \mathrm{CO}_{2}$ for 30 minutes, followed by addition of $3 \mathrm{ml}$ of Dulbecco's modified Eagle's medium (DMEM, Solarbio, Beijing, China). After precipitation, the supernatant was obtained and filtered through a cell sieve. The cells were then seeded into 6-well plates at an adjusted concentration of $5 \times 10^{5} / \mathrm{ml}$, followed by incubation at $37^{\circ}$ with $5 \% \mathrm{CO}_{2}$ for $6 \mathrm{~h}$. Afterwards, the DMEM was replaced with Neurobasal-A medium (10888022, Thermo Fisher Scientific, USA), and the neurons were incubated for another 7 days [20].

\section{Neuron morphological identification and immunofluorescence staining}

Neuron morphological identification was performed on days 3, 5 and 7 by using an optical microscope (BX53M, Olympus, Japan). After the neurons were incubated for $72 \mathrm{~h}$, they were washed with PBS (0.01 mol/l, pH 7.4) three times, fixed with $4 \%$ paraformaldehyde for $20 \mathrm{~min}$, and permeated with $0.1 \%$ 
TritonX-100 (X100, Sigma-Aldrich, USA) for $30 \mathrm{~min}$. Subsequently, $5 \%$ albumin from bovine serum (BSA; \#9998, Cell Signaling Technology, USA) was employed for sealing for $20 \mathrm{~min}$. Next, the neurons were incubated with primary antibody anti-MAP-2 antibody (ab183830, $1: 200$, Abcam, UK) overnight at 4\%, followed by $1 \mathrm{~h}$ incubation with the secondary antibody goat anti-rabbit lgG H\&L (ab150077, 1 : 500, Abcam, UK) at $37^{\circ}$ without light. Before sealing with glycerine, $1 \mathrm{\mu g} / \mathrm{ml}$ of DAPI staining solution (C1005, Beyotime, China) was added to incubate the neurons for $4 \mathrm{~min}$. The neuron growth states were analysed via ImageJ (version 5.0; Bio-Rad, Hercules, CA, USA).

\section{Transfection}

The miR-1b mimic ( $M$; sequence: 5'-UGGAAUGU AAAGAAGUAUGUAU-3'), miR-1b inhibitor ( 1 ; sequence: 5'-AUACAUACUUCUUUACAUUCCA-3'), and their negative controls (mimic control - MC, inhibitor control - IC) were purchased from GenePharma (Shanghai, China) and then transfected into neurons using Lipofectamine 2000 Transfection Reagent (Invitrogen, USA) in strict accordance with the manufacturer's protocols.

Meanwhile, the small interfering RNA for KLF7 (siKLF7) and negative control siRNA (siNC) were obtained from RiboBio Co., Ltd. (Guangzhou, China) for this study, and pcDNA3.1 plasmid carrying overexpressed KLF7 was purchased (VT1010, YouBio, China). They were transfected into neurons $\left(2.5 \times 10^{3}\right.$ cells per well) using Lipofectamine RNAiMAX Transfection Reagent (Thermo Fisher Scientific, USA) following the manufacturer's instructions. Neurons transfected with empty plasmid were used as the control group. In this paper, the siRNA sequences used for KLF7 were listed below: KLF7 siRNA: sense 5'-GCCAACCAGCUCUUCUCUATT-3', antisense 5'-UAGAGAAGAGCUGGUUGGCTT-3'; si-negative control (siNC): sense 5'-UUCUCCGAACGUGUCACGUTT-3', antisense 5'-ACGUGACACGUUCGGAGAATT-3'. After transfection for $48 \mathrm{~h}$, cells were collected for subsequent analysis.

\section{RNA extraction and real-time quantitative PCR (qRT-PCR)}

Total RNA was extracted from the neurons by using the TRlzol reagent (D9108A, Takara Bio) based on the manufacturer's instructions. RNA concentration was quantified with a spectrophotometer (ND-LITE, Automation Technologies Online, Thermo Fisher Scientific,
USA). The mRNA expression of KLF7 was measured with a SYBR Premix Ex Taq ${ }^{\text {TM }}$ kit (Takara, Dalian, China). For miR-1b expression detection, a miRcute Plus miRNA qPCR Detection Kit (Tiangen, China) was applied. All RT-PCR reactions were conducted on the ABI PRISM 7500 System (Applied Biosystems, Foster City, (A) with the following parameters: $95^{\circ} \mathrm{C}$ for $5 \mathrm{~min}, 40$ cycles of $95^{\circ} \mathrm{C}$ for $15 \mathrm{~s}, 60^{\circ} \mathrm{C}$ for $30 \mathrm{~s}$, and $70^{\circ} \mathrm{C}$ for $10 \mathrm{~s}$. GAPDH (for KLF7) and U6 (for miR-1b) were used as internal references. The primer sequences in this study were listed in Table I. The relative gene expression was analysed via the $2^{-\Delta \Delta C t}$ method [21].

\section{MTT assay}

Neuron viability was detected via MTT assay. Firstly, neurons $\left(1 \times 10^{4}\right.$ cells/well) were seeded in a 384-well plate, and then $36 \mu \mathrm{l}$ of MTT reagent (A13261, Thermo Fisher Scientific, USA) was added into each well. Subsequently, the neurons were incubated at $37^{\circ}$ with $5 \% \mathrm{CO}_{2}$ overnight. And then $1.5 \mathrm{ml}$ of dimethyl sulfoxide (DMSO) was employed to dissolve the formazan. A multifunction enzyme-linked analyser (Varioskan LUX, Thermo Fisher Scientific, USA) was used to detect the absorbance value OD at $490 \mathrm{~nm}$ for 4 hours.

\section{Flow cytometry}

Neuron apoptosis was measured by flow cytometry using the Annexin V-FITC Apoptosis Detection Kit (BMS500FI, Thermo Fisher Scientific, USA). After transfection, neurons were washed with cold PBS three times, followed by 48 -hour incubation. Subsequently, the neurons were supplemented with $5 \mu \mathrm{l}$ of Annexin $V$ and $5 \mu \mathrm{l}$ of propidium iodide (PI) together

Table I. Primer for qRT-PCR

\begin{tabular}{lc}
\hline Gene & \multicolumn{1}{c}{ Primers } \\
\hline \begin{tabular}{l} 
MiR-1b \\
\hline Forward
\end{tabular} & 5'-ATGTCGTATCCAGTGCAATTGC-3' \\
\hline Reverse & 5'-GTCGTATCCAGTGCGTGTCG-3' \\
\hline KLF7 & \\
\hline Forward & 5'-TTTCCTGGCAGTCATCTGCAC-3' \\
\hline Reverse & 5'-GGGTCTGTTTGTTTGTCAGTCTGTC-3' \\
\hline GAPDH & 5'-GGAGAGTTTTTGGTTTTAGGGTTAG-3' \\
\hline Forward & 5'-AAAACCTCCTATAATATCCCTCCTC-3' \\
\hline Reverse & \\
\hline U6 '-CCATCGGAAGCTCGTATACGAAATT-3' \\
\hline Forward & 5'-GGCCTCTCGAACTTGCGTGTCAG-3' \\
\hline Reverse
\end{tabular}


without light for 15 minutes at room temperature. A flow cytometer (ACEA NovoCyte, ACEA Biosciences, Inc., USA) was applied to detect neuron apoptosis.

\section{Western blot}

The neurons were lysed using RIPA buffer (\#9806, Cell Signaling Technology, Danvers, MA, USA). Protein concentration was determined with a Bicinchoninic protein assay (BCA) kit (7780S, Cell Signaling Technology, Danvers, MA, USA). All the protein samples were then electrophoresed via 10\% SDS-PAGE (P0012A Beyotime, China). Later, the samples were transferred to polyvinylidene fluoride membranes (PVDF; Millipore, Bedford, MA) which were blocked by $5 \%$ skimmed milk at room temperature for $60 \mathrm{~min}$. The membranes were then incubated with the following primary antibodies overnight at $4^{\circ} \mathrm{C}$ : anti-GAPDH antibody (rabbit, \#5174, $1: 1000$, Cell Signaling Technology, USA), anti-NGF antibody (rabbit, ab221609, 1 : 1000, Abcam, UK), anti-C-caspase-3 antibody (rabbit, ab2302, $1 \mu \mathrm{g} / \mathrm{ml}$, Abcam, UK), antiCNTF antibody (rabbit, ab46172, $1:$ 3000, Abcam, UK) and anti-BDNF antibody (rabbit, ab108319, $1: 1000$, Abcam, UK). The membranes were then incubated with the horseradish peroxidase (HRP)-conjugated secondary antibody goat anti-rabbit IgG H\&L (HRP) (ab205718, $1: 2000$, Abcam, UK) for $1 \mathrm{~h}$ at room temperature and washed with tris-buffer saline tween (TBST) three times. GAPDH was employed as the normalization reference. An enhanced chemiluminescence (ECL) plus kit (K22030, Abbkine Scientific, China) was employed to measure the labelled proteins [36].

\section{Prediction of target gene and dual-luciferase reporter assay}

Target genes and potential binding sites of KLF7 and miR-1b were predicted by TargetScan V7.2 (http://www.targetscan.org/vert 72/) and then confirmed by dual-luciferase reporter assay.

PMIR-REPORT Luciferase vectors (AM5795; Thermo Fisher Scientific, USA) which contained KLF7 sequences (wild-type or mutated) were cloned into the pMirGLO reporter vector (Promega, Madison, WI, USA) to construct KLF7-WT and KLF7-MUT. Neurons $\left(5 \times 10^{3}\right.$ cells/well) were incubated in 96-well plates, and then luciferase reporter plasmids were co-transfected with miR-1b mimic (M), inhibitor (I) or their respective controls (mimic control - MC, inhibitor control - IC) into the neurons using Lipofectamine 2000 Transfection reagent (Thermo Fisher Scientific,
Waltham, MA, USA). Luciferase detection was conducted in the dual-luciferase report assay system (E1910; Promega, USA) and the luciferase activity was normalized to Renilla luciferase activity.

\section{Statistical analysis}

Values were represented as mean \pm SD. Statistical analysis was performed via the SPSS software (version 22.0, SPSS Inc., Chicago, IL, USA). Multiple comparisons were performed using one-way ANOVA, followed by a post hoc test. $P$-values of less than 0.05 were considered statistically significant.

\section{Results \\ Primary culture and characterization of rat neurons}

After the neurons were successfully cultured, neuron morphological identification was performed using an optical microscope. On the third day, neuronal synapses were increased, and neural networks were gradually formed; on day 5 , connections in neural networks were formed in synapses; and on day 7 , the neurons were plump and grew into mature neurons (Fig. 1A). Microtubule-associated protein 2 (MAP-2) was a marker of neuronal differentiation, and its function was regulated by phosphorylation both during neuronal development and after differentiation during synaptic activity for maintenance of neuronal health [28]. The results of MAP-2 immunofluorescence staining and DAPI nuclear staining showed that neuron cell body and axon exhibited good morphology and the neurons were of high purity (Fig. 1B).

\section{MiR-1b regulated neuron viability and regenerative ability}

To discover the different expression of miR-1b in neurons, the neurons were transfected with miR-1b mimic (M) or inhibitor (I), and the corresponding control groups ( $M C$ and IC) were set up at the same time. As revealed in Figure $2 \mathrm{~A}$, the mRNA expression of miR-1b in the $M$ group was higher in comparison with the $M C$ group ( $p<0.001$ ), suggesting that overexpression of miR-1b was successfully transfected in neurons. In addition, the results of MTT assay demonstrated that neuron viability in the $M$ group was lower than that in the $M C$ group (Fig. $2 B, p<0.001$ ). On the contrary, neuron viability in the I group was higher when compared with the IC group (Fig. 2B, $p<0.01$ ), indicating 
A
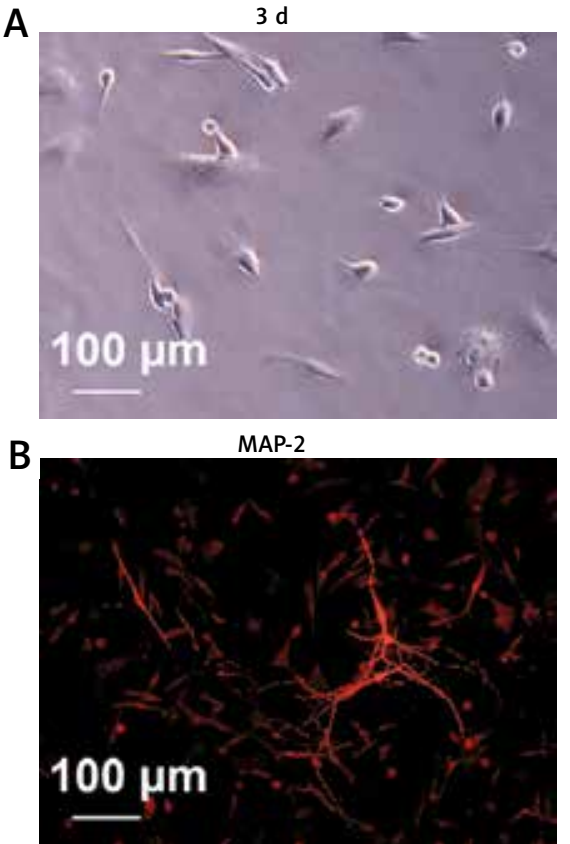

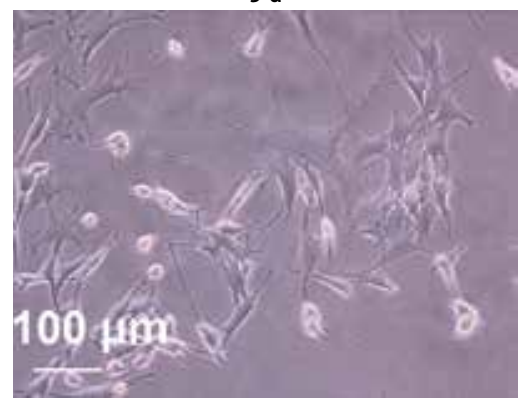

DAPI

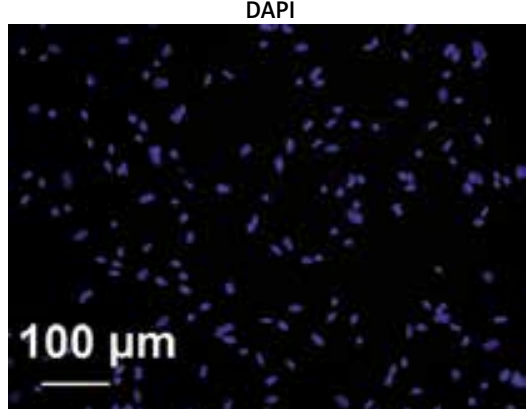

$7 d$

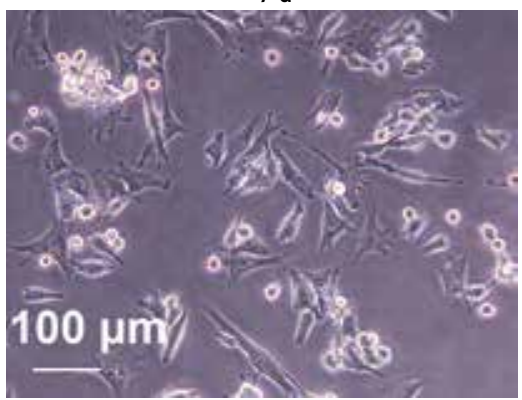

Merge

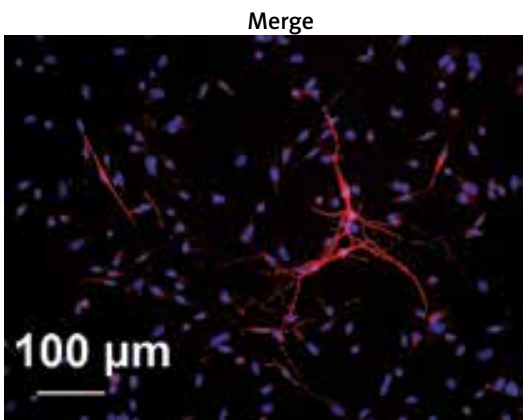

Fig. 1. Primary culture of rat neurons was successfully conducted. A) Rat neuron morphological identification was performed using a microscope. Magnification: 200x, scale bar $=100 \mu \mathrm{m}$. B) Neuronal identification was carried out by microtubule-associated protein 2 (MAP-2) immunofluorescence staining and DAPI nuclear staining. Magnification: 200x, scale bar $=100 \mu \mathrm{m}$. All experiments have been performed in triplicate and experimental data were expressed as mean \pm standard deviation (SD).

that neuron viability was down-regulated via up-regulation of miR-1b, which was rescued by low expression of miR-1b.

Besides, neuron regenerative ability was investigated using a microscope and the Image J software after 48 hours of incubation. As shown in Figure 2C-E, the length of neurites per cell, the percentage of cells with neurites and the number of branches per cell in the $M$ group were decreased in comparison with the MC group ( $p<0.05)$. Interestingly, the above indicators in the I group were increased when compared with the IC group (Fig. 2C-E, $p<0.05$ ). These data demonstrated that neuron regenerative ability was promoted by up-regulation of miR-1b, which was rescued by low expression of miR-1b.

\section{MiR- $1 \mathrm{~b}$ regulated the neuron apoptosis and regeneration-related proteins expressions}

After transfection, neuron apoptosis was detected through flow cytometry. It was clear that the apoptosis rate in the $M$ group was significantly enhanced when compared with the MC group, while in the I group, it was lowered as compared with the IC group (Fig. 3A, $p<0.001$ ).

Expressions of cell regeneration-related proteins in the transfected neurons were measured via Western blot. As shown in Figure 3B, the expressions of nerve growth factor (NGF), ciliary neurotrophic factor (CNTF) and brain-derived neurotrophic factor (BDNF) in the $M$ group were lower than those in the $M C$ group, while these protein expressions in the I group were promoted when compared with the IC group $(p<0.05)$. Meanwhile, cleaved (C)-caspase-3 expression was up-regulated in the $M$ group in comparison with the MC group, but it was down-regulated in the I group when compared with the IC group (Fig. 3B, $p<0.001$ ), suggesting that up-regulation of miR-1b decreased the expressions of proteins related to cell regeneration but increased C-caspase- 3 expression, which was rescued by low expression of miR-1b.

\section{KLF7 was the target of miR-1b}

The prediction software TargetScan V7.2 showed that KLF7 was a target of miR-1b, with the target site located in the 3'-UTR (Fig. 4A). With the aim to further validate that miR-1b directly binds to KLF7, the 
A
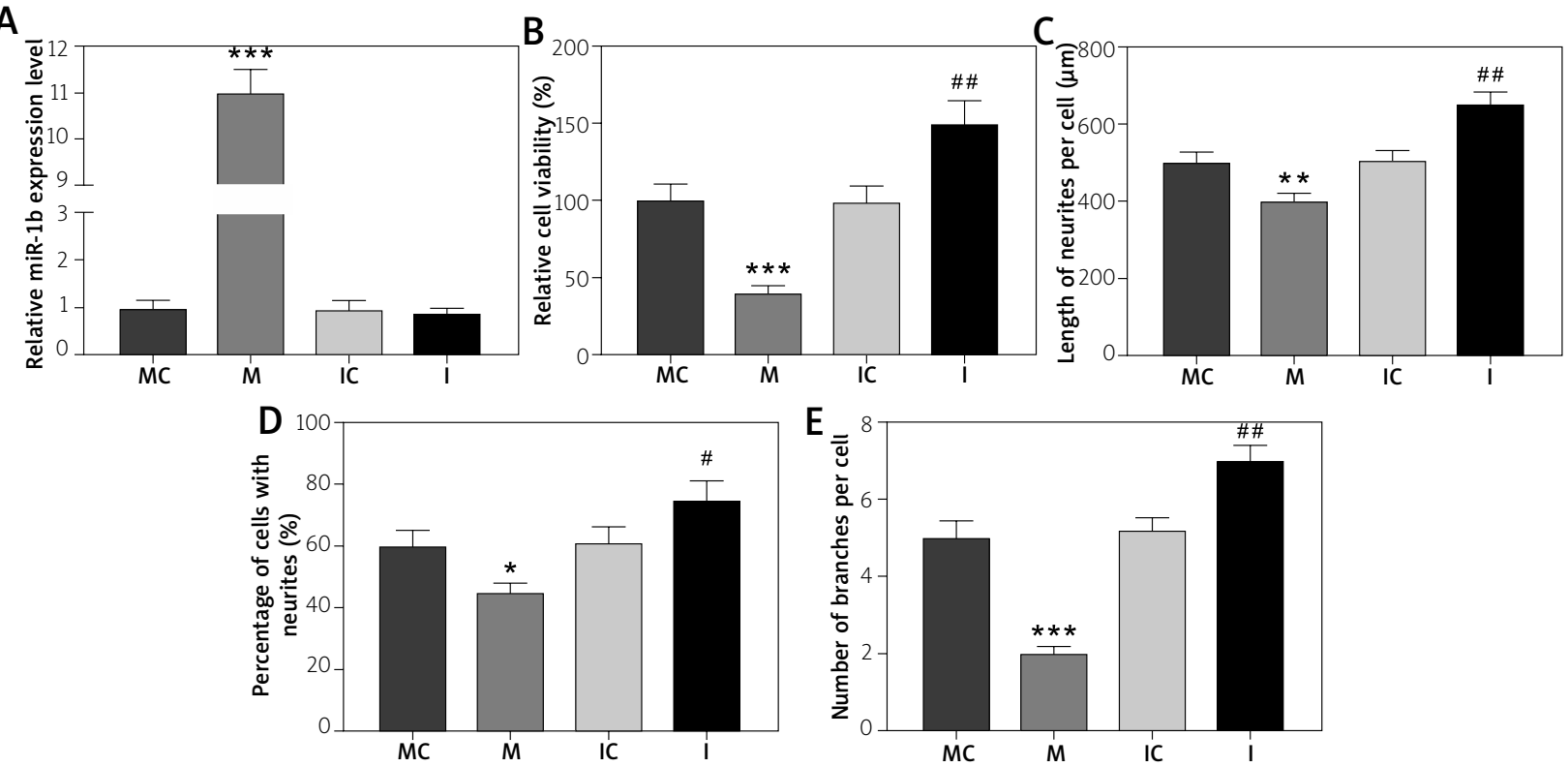

Fig. 2. MiR-1b regulated neuron viability and regenerative ability. A) The transfection rate of miR-1b in neurons was measured by quantitative real-time polymerase chain reaction (qRT-PCR). U6 was used as the internal reference. B) After transfection, neuron viability was detected through MTT assay. C-E) The length of neurites per cell, the percentage of cells with neurites and the number of branches per cell after transfection were investigated using a microscope and the Image J software. All experiments have been performed in triplicate and experimental data were expressed as mean \pm standard deviation (SD). ${ }^{*} p<0.05,{ }^{* *} p<0.01,{ }^{* *} p<0.001$, vs. MC; ${ }^{\#} p<0.05,{ }^{\# \#} p<0.01$, vs. IC. MC - mimic control, IC - inhibitor control, $M-$ miR-1b mimic, I - miR-1b inhibitor.

dual luciferase reporter assay was performed. It was discovered that the luciferase activity was reduced in the KLF7-WT-mimic group in comparison with the MC group, while it was enhanced in the KLF7-WTinhibitor group when compared with the IC group (Fig. 4B, C, $p<0.01$ ), indicating that KLF7 was targeted by miR-1b. Moreover, the results of qRT-PCR showed that miR-1b mimic mitigated the up-regulation of the KLF7 level after transfection of overexpressed KLF7 plasmid, and miR-1b inhibitor rescued the down-regulation of KLF7 level after knockdown of KLF7, which further proved that KLF7 was the target gene of miR-1b (Fig. 4D-G, $p<0.001$ ).

\section{Effects of miR-1b on neuron viability, regeneration and apoptosis through targeting KLF7}

The outcomes of MTT assay revealed that the viability of neurons transfected with overexpressed KLF7 plasmid was promoted compared with the negative control (NC) group (Fig. 5A, $p<0.01$ ). Besides, co-transfection of overexpressed KLF7 plasmid and miR-1b mimic weakened neuron viability compared with the KLF7 group (Fig. 5A, $p<0.01$ ). Conversely, the viability of neurons transfected with specific siRNA against KLF7 or co-transfected with miR-1b inhibitor and specific siRNA against KLF7 displayed an opposite result (Fig. 5B, $p<0.05$ ). These findings demonstrated that overexpressed KLF7 partially rescued the effects of up-regulation of miR-1b on neuron viability.

Through microscope observation and Image J software analysis, it was found that the length of neurites per cell, the percentage of cells with neurites and the number of branches per cell after transfection of overexpressed KLF7 plasmid were evidently promoted in comparison with the NC group (Fig. 5C-E, $p<0.05$ ). Meanwhile, these regeneration indicators were up-regulated via co-treatment with overexpressed KLF7 plasmid and miR-1b mimic compared with the $M$ group (Fig. $5 C-E, p<0.05$ ). By contrast, transfection of specific siRNA against KLF7 or co-transfection of miR-1b inhibitor and specific siRNA against KLF7 into neurons was counter-productive (Fig. 5F-H, $p<0.05$ ). These data proved that overexpressed KLF7 partially rescued the effects of up-regulation of miR-1b on neuron regeneration. 

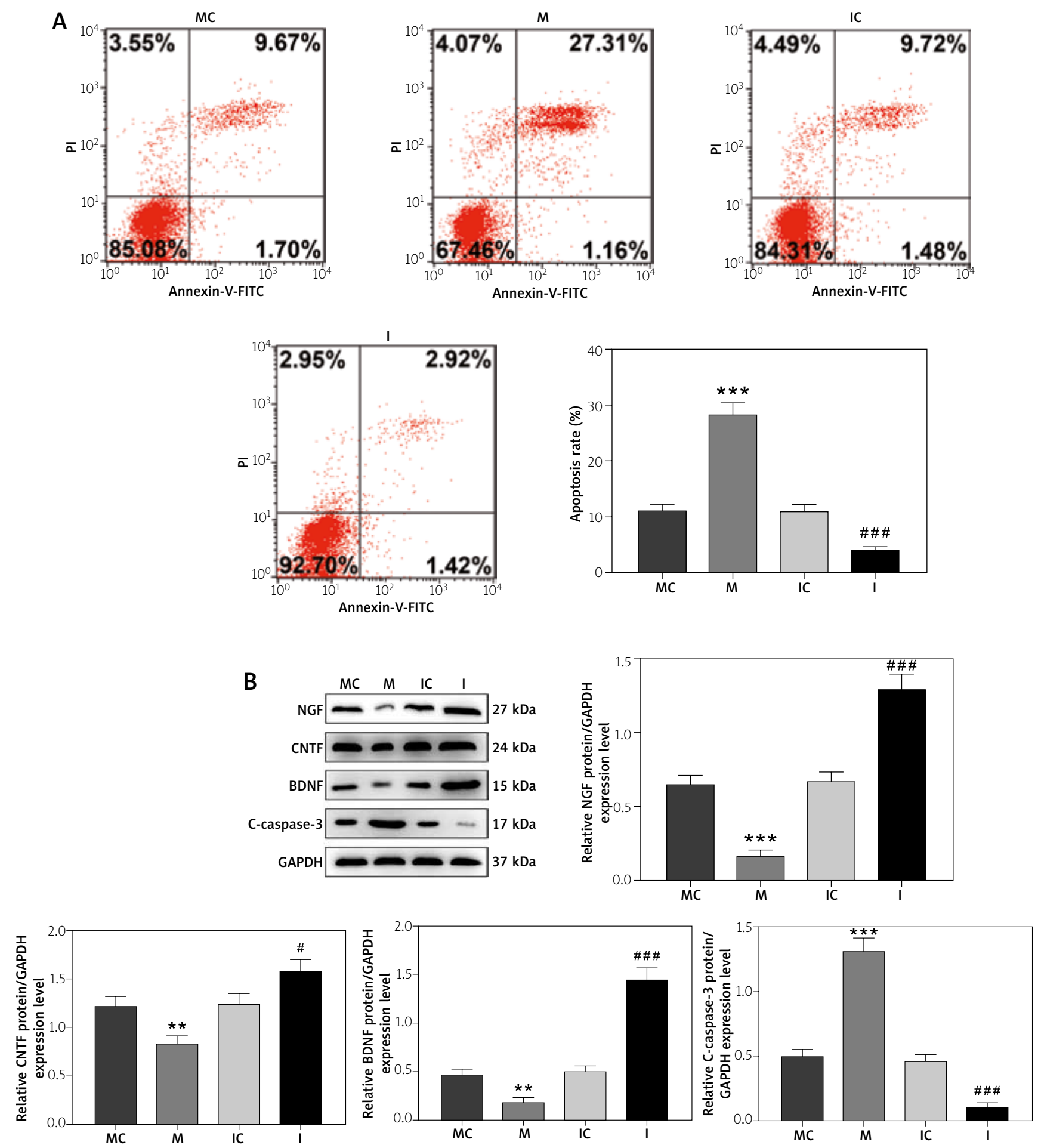

Fig. 3. MiR-1b regulated neuron apoptosis and the expressions of regeneration-related proteins. A) Neuron apoptosis after transfection was measured by flow cytometry. B) Expressions of nerve growth factor (NGF), ciliary neurotrophic factor (CNTF), brain-derived neurotrophic factor (BDNF) and C-caspase-3 after transfection were detected through Western blot. GAPDH was used as the internal reference. All experiments have been performed in triplicate and experimental data were expressed as mean \pm standard deviation (SD). ${ }^{* *} p<0.01,{ }^{* * *} p<0.001$, vs. MC;

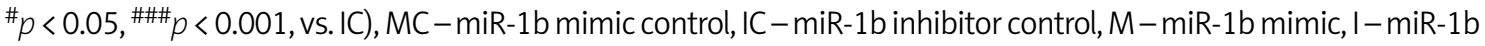
inhibitor. 
A

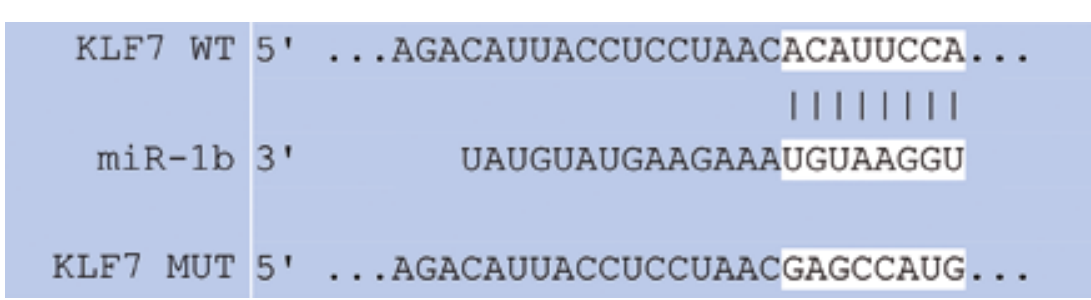

B
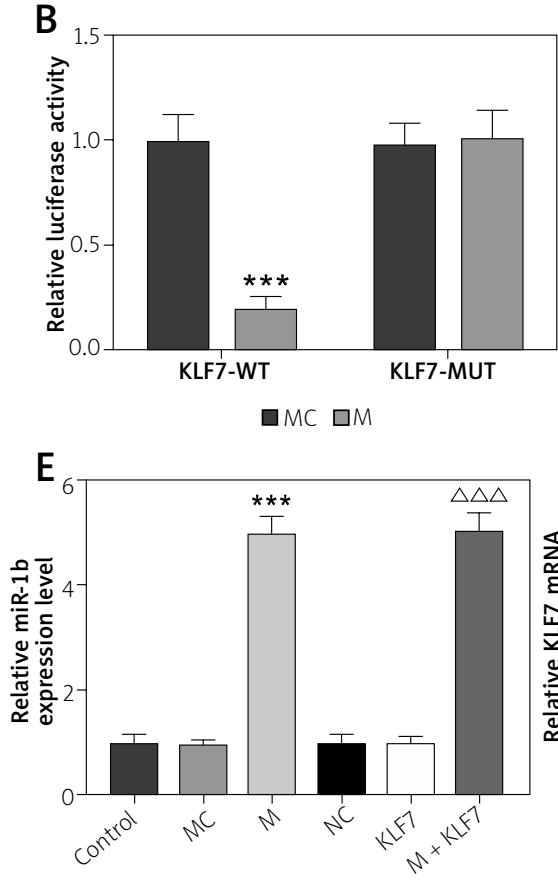

C

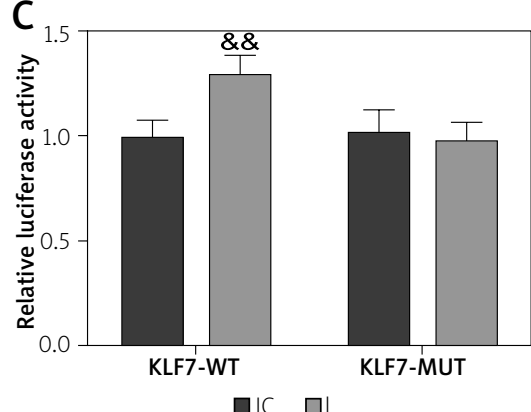

미미

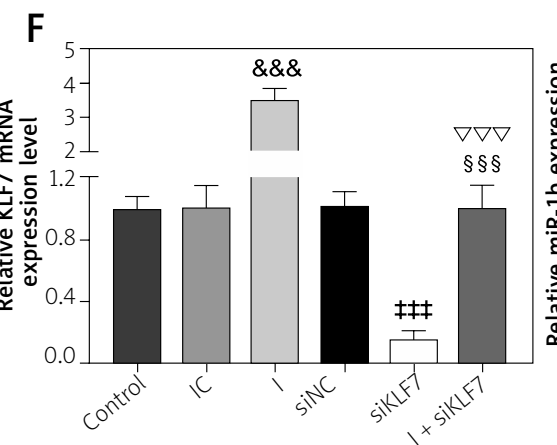

D

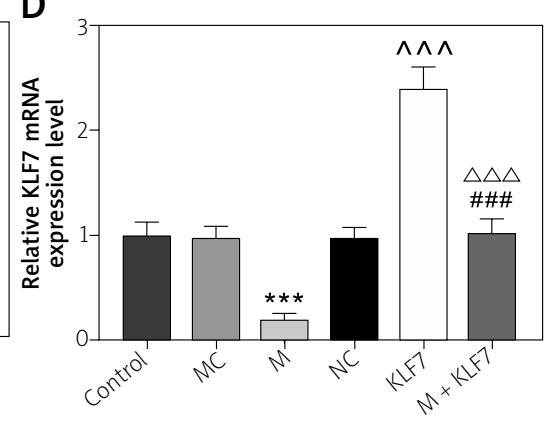

G

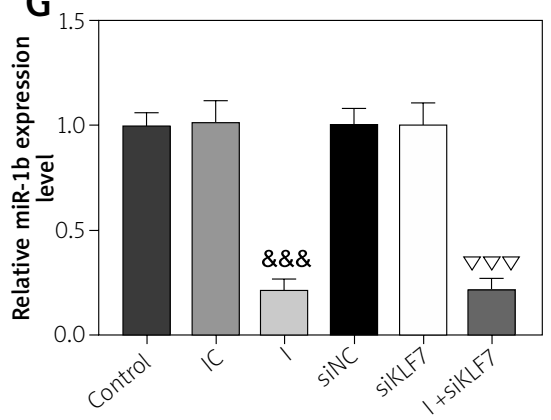

Fig. 4. KLF7 was the target of miR-1b. A) TargetScan V7.2 was used to predict the binding sites of miR-1b and KLF7. B, C) Wild-type or mutant KLF7 (KLF7-WT, KLF7-MUT) was separately co-transfected with miR-1b mimic and miR-1b inhibitor into the neuron, and luciferase activity was measured by dual-luciferase reporter assay. D-G) The mRNA expressions of KLF7 and miR-1b in neurons after transfection were detected by qRT-PCR. GAPDH (for KLF7) and U6 (for miR-1b) were used as internal references. All experiments have been performed in triplicate and experimental data were expressed as mean \pm standard deviation (SD). ${ }^{* * *} p<0.001$, vs. MC;

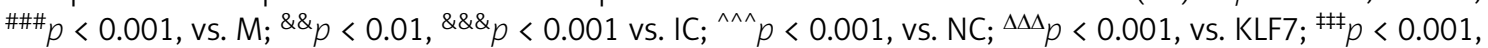
vs. siNC; ${ }^{\nabla \nabla} p<0.001$, vs. siKLF7; ${ }^{\S \S} p<0.001$, vs. I. KLF7 - Krüppel-like factor 7, M - miR-1b mimic, I - miR$1 b$ inhibitor, $M C$ - miR-1b mimic control, IC - miR-1b inhibitor control, NC - negative control, siKLF7 - small interfering RNA for KLF7 (siKLF7), siNC - negative control for small interfering RNA for KLF7.

In addition, the results of flow cytometry demonstrated that neuron apoptosis was significantly reduced after transfection of overexpressed KLF7 plasmid compared with the NC group (Fig. 6A, $p<0.001$ ). Co-transfection of overexpressed KLF7 plasmid and miR-1b mimic mitigated neuron apoptosis in comparison with the M group. However, the apoptosis rates of neurons transfected with specific siRNA against KLF7 or co-transfected with miR-1b inhibitor and specific siRNA against KLF7 exhibited an opposite result (Fig. 6B, $p<0.001)$. The results herein were sufficient to support that miR-1b regulated neuron viability, regeneration and apoptosis through targeting KLF7.

\section{Effects of miR-1b on the expressions of C-caspase- 3 and neuron regeneration- related proteins through targeting KLF7}

The results of Western blot proved that the expressions of NGF, CNTF and BDNF were evidently increased yet C-caspase-3 expression was 

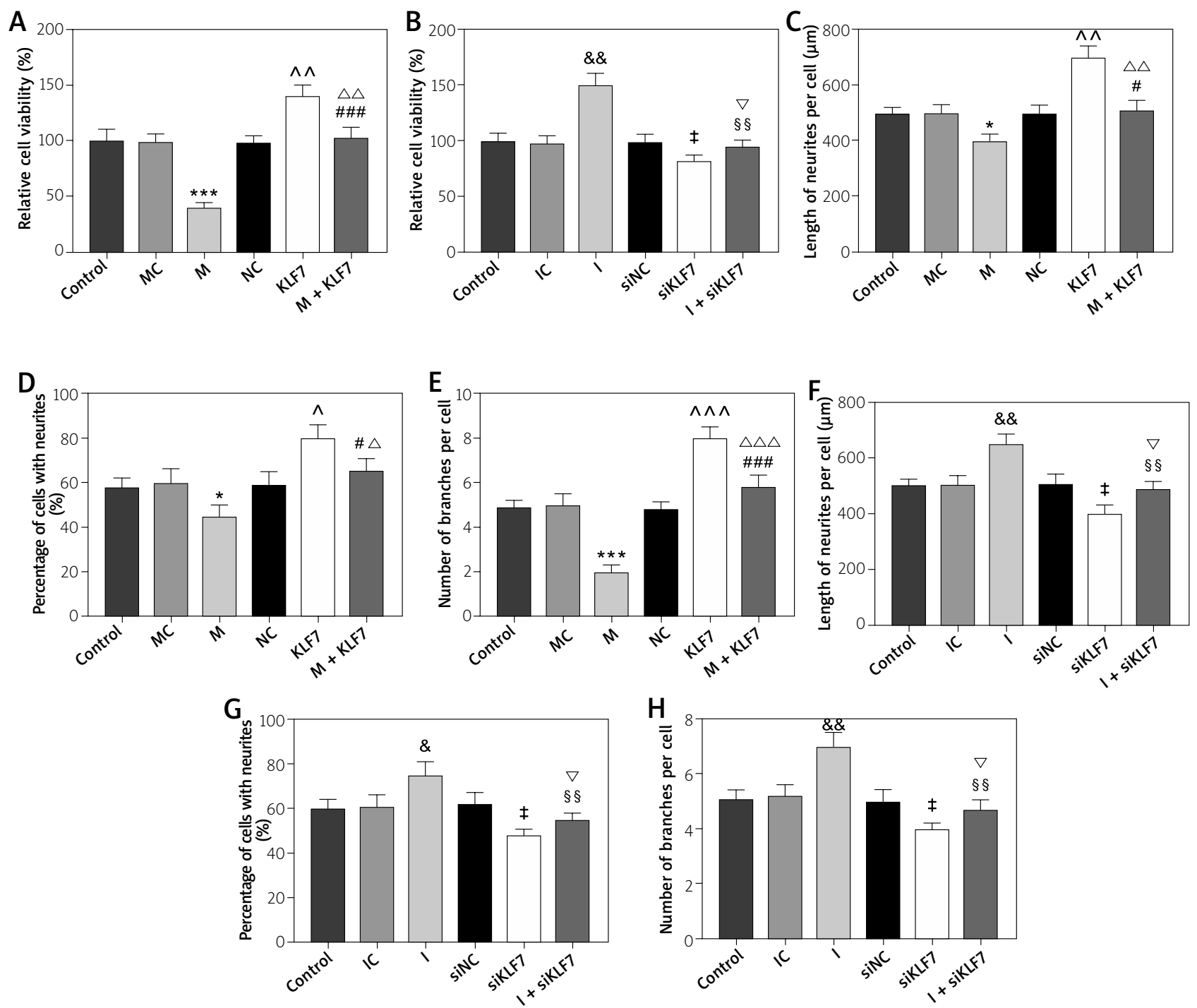

Fig. 5. Effects of miR-1b on neuron viability and regeneration through targeting KLF7. A, B) Inhibitory effect of overexpressed miR-1b on neuron viability via targeting KLF7 was detected by MTT assay, and low expressions of miR-1b and KLF7 performed the opposite function. C-E) Suppressive role of overexpressed miR-1b in the length of neurites per cell, the percentage of cells with neurites and the number of branches per cell via targeting KLF7 was investigated using a microscope and ImageJ. F-H) Promoting role of down-regulation of miR-1b on the length of neurites per cell, the percentage of cells with neurites and the number of branches per cell via targeting KLF7 was determined using a microscope and ImageJ. All experiments have been performed in triplicate and experimental data were expressed as mean \pm standard deviation (SD). ${ }^{*} p<0.05$, ${ }^{* *} p<0.001$, vs. MC; ${ }^{\wedge} p<0.05,{ }^{\wedge} p<0.01,{ }^{\wedge \wedge \wedge} p<0.001$, vs. NC; ${ }^{\Delta} p<0.05,{ }^{\Delta \Delta} p<0.01,{ }^{\Delta \Delta \Delta} p<0.001$, vs. KLF7; ${ }^{\#} p<0.05,{ }^{\# \#} p<0.001$, vs. M; ${ }^{*} p<0.05, \& \& p<0.01$, vs. IC; ${ }^{\ddagger} p<0.05$, vs. siNC; ${ }^{\nabla} p<0.05$, vs. siKLF7; ${ }^{\S} p<0.01$, vs. I.

decreased in the neurons that were transfected with overexpressed KLF7 plasmid compared with the NC group (Fig. 7A, $p<0.01$ ). In addition, co-transfection of overexpressed KLF7 plasmid and miR-1b mimic facilitated the expressions of NGF, CNTF and BDNF while mitigating C-caspase-3 expression compared with the $M$ group (Fig. 7A, $p<0.05$ ). On the contrary, the expressions of NGF, CNTF, BDNF and C-caspase-3 in neurons that were transfected with specific siRNA against KLF7 or co-transfected with miR-1b inhibitor and specific siRNA against KLF7 showed an opposite result (Fig. 7B, $p<0.01$ ). These data revealed that 

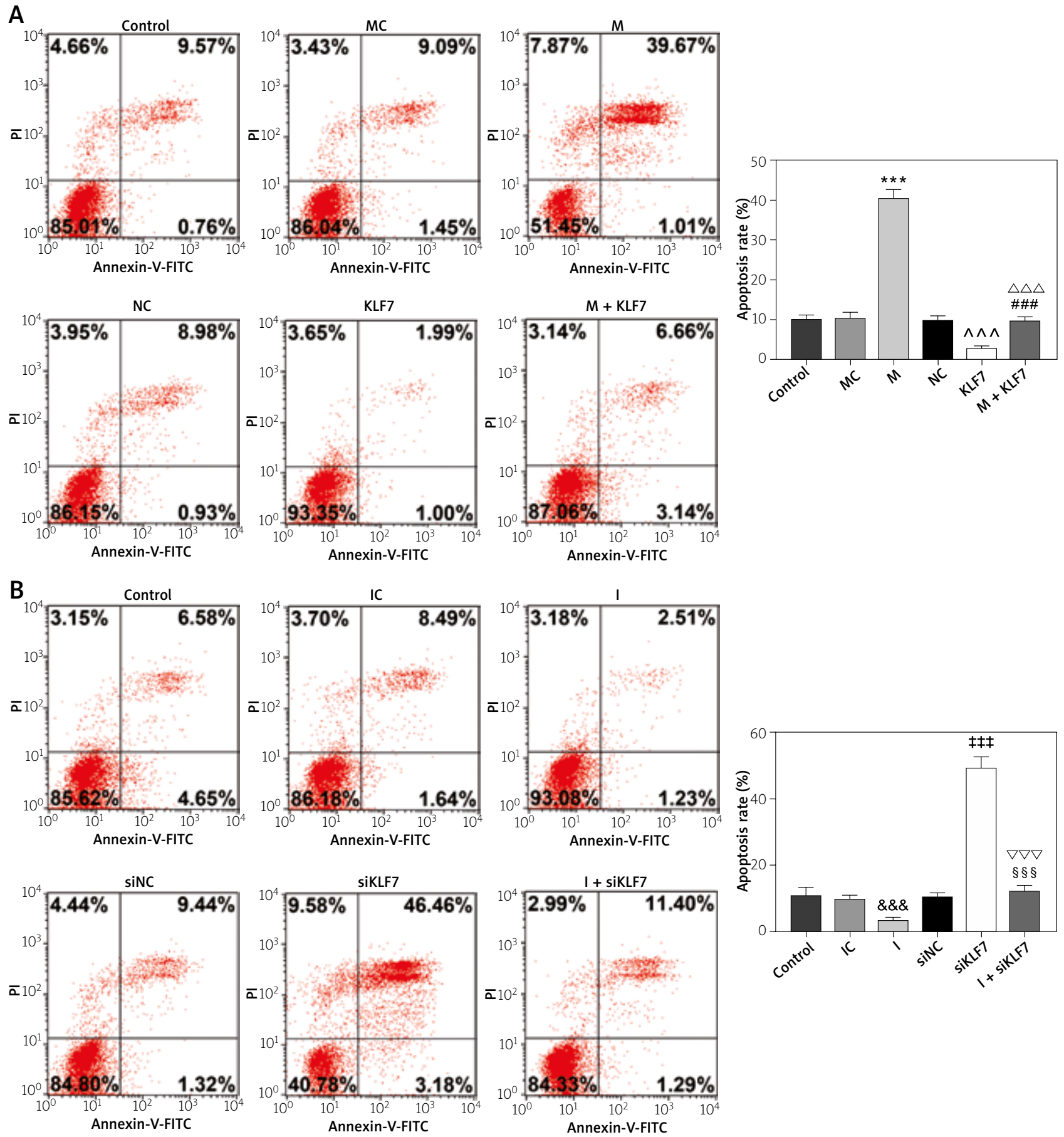

Fig. 6. Effects of miR-1b on neuron apoptosis through targeting KLF7. A) Positive effect of overexpressed miR-1b on neuron apoptosis via targeting KLF7 was measured through flow cytometry. B) Repressive effect of low expression of miR-1b on neuron apoptosis via regulating KLF7 was identified by flow cytometry. All experiments have been performed in triplicate and experimental data were expressed as mean \pm stan-

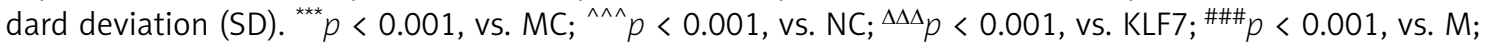
$\& \& \& p<0.001$, vs. IC; ${ }^{\sharp \neq} p<0.001$, vs. siNC; ${ }^{\nabla \nabla \nabla} p<0.001$, vs. siKLF7; $\$ \S \S<0.001$, vs. I. 

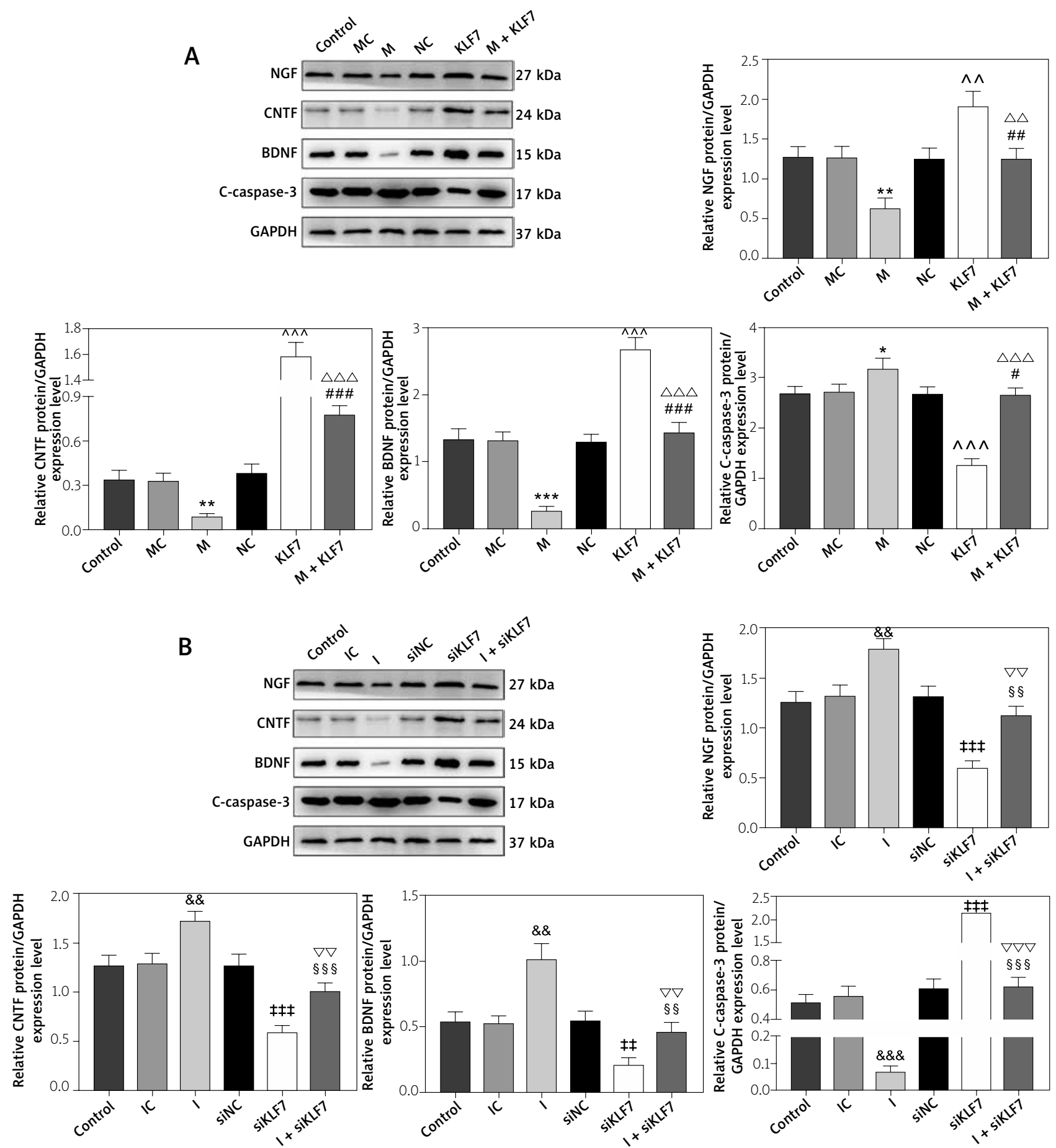

Fig. 7. Effects of miR-1b on the expressions of C-caspase-3 and neuron regeneration-related proteins through targeting KLF7. A) Effect of overexpressed miR-1b on inhibiting the expressions of nerve growth factor (NGF), ciliary neurotrophic factor (CNTF) and brain-derived neurotrophic factor (BDNF) and promoting cleaved (C)-caspase-3 expression via targeting KLF7 was detected by Western blot. GAPDH was used as the internal reference. B) Effect of low expression of miR-1b on promoting the expressions of NGF, CNTF and BDNF but suppressing C-caspase-3 expression via targeting KLF7 was investigated by Western blot. GAPDH was used as the internal reference. All experiments have been performed in triplicate and experimental data were expressed as mean \pm standard deviation (SD). ${ }^{*} p<0.05,{ }^{* *} p<0.01,{ }^{* * *} p<0.001$, vs. MC; ${ }^{\wedge} p<0.01,{ }^{\wedge \wedge} p<0.001$, vs. NC; ${ }^{\Delta \Delta} p<0.01,{ }^{\Delta \Delta \Delta} p<0.001$, vs. KLF7; ${ }^{\#} p<0.05,{ }^{\# \#} p<0.01,{ }^{\# \#} p<0.001$, vs. M; \&\& $p<0.01$, \&\&\& $p<0.001$, vs. IC; ${ }^{\# \#} p<0.01,{ }^{\# \#} p<0.001$, vs. siNC; $\nabla \nabla p<0.01,{ }^{\nabla \nabla \nabla} p<0.001$, vs. siKLF7; ${ }^{\S} p<0.01,{ }^{\S \S} p<0.001$, vs. I. 
overexpressed KLF7 partially rescued the effects of up-regulation of miR- $1 \mathrm{~b}$ on the expressions of C-caspase-3 and neuron regeneration-related proteins.

\section{Discussion}

Recovery from central nervous system (CNS) injury is limited by the inability of axons to regenerate and rebuild effective communication [33]. Besides, axon growth can be promoted via targeting the intrinsic growth capacity of neurons through forced expression of regeneration-associated transcription factors [2]. In addition, increasing evidence has shown that a great number of miRNAs are involved in neuron generation via regulating their target genes, which may be transcription factors [10,22].

For figuring out the regulatory role of miRNAs in neurons of the brain, it is essential to conduct neuron-specific analysis of miRNAs and their target genes. It has been reported that miR-1 was down-regulated following sciatic nerve injury that increased the expression and secretion of BDNF, which benefits nerve growth and regeneration [34]. Neumann et al. discovered that miR-1 expression in the sciatic nerve was time-dependently decreased and the protein expressions of the miR-1 targets BDNF and Connexin 43 were upregulated in the sciatic nerve and dorsal root ganglion [27]. MiR-1b is a member of the miR-1 family with a highly homologous sequence to miR-1 [20]. In our study, miR-1b mimic and inhibitor were transfected into primarily cultured neurons, and consistent with previous findings, we discovered that a low expression of miR-1b rescued the reduction of neuron viability and regenerative ability caused by up-regulation of miR-1b [20].

Nerve growth factor is a well-studied neurotrophic factor and one of the first identified growth factors [11]. Ciliary neurotrophic factor is a potent inducer of axonal regeneration [12]. Moreover, BDNF is a member of the neurotrophin family of secreted proteins [29] and Caspase-3 is a major mediator of activated apoptosis [35]. According the results of Western blot in our study, the expressions of NGF, CNTF and BDNF were down-regulated but cleaved (C)-caspase-3 expression was up-regulated in neurons by overexpression of miR-1b, which further proved that overexpression of miR-1b inhibited neuron regeneration.

The Krüppel-like transcription factors (KLFs) are important regulators of cell proliferation and differ- entiation as well as embryonic development [14]. More recently, KLFs have proved as important regulators of the neuron-intrinsic capacity for regeneration, with various members displaying either anti- or pro-regenerative roles [32]. For example, knocking out KLF4 enhanced axon growth and regeneration in developing retinal ganglion cells, and KLF4 acted independently on cell survival to suppress axon and dendrite initiation and elongation in hippocampal neurons in vitro [25]. The inhibition of miR-124 expression promoted motor neuron regeneration through targeting KLF6 and STAT3 [26]. KLF7, a member of the KLFs family, has been described as a regulator of axon outgrowth [31]. Moreover, Kajimura et al. demonstrated that loss of KLF7 primarily affects the activity of genes involved in olfactory sensory neuron differentiation, axonal growth, cytoskeletal dynamics, cell adhesion and synaptogenesis [13]. In central nervous system and peripheral nerve injury, KLF7 plays a positive role through promoting growth, axonal regeneration, and sprouting in injured nerves $[3,16]$. Importantly, KLF7 has been reported to be the target gene of miR-146b, and inhibition of miR-146b promotes sciatic nerve regeneration via regulating KLF7 [17], suggesting that KLF7 may be beneficial to neuron regeneration. However, whether KLF7 was the target of miR-1b and the interaction of KLF7 and miR-1b in neurons remain elusive.

In our study, we further identified that KLF7 was the target gene of miR-1b. Our experimental results uncovered that overexpressed KLF7 rescued the effects of up-regulating miR-1b on neuron viability, regeneration and apoptosis, and also partially rescued the effect of up-regulating miR-1b on the expressions of NGF, CNTF, BDNF and C-caspase-3. However, the mechanism by which KLF7 played its role in regulating neuron apoptosis and regeneration-related proteins was not well illuminated, which was a limitation in our study. It was reported that KLF7 may directly regulate TrkA, which is a crucial nerve growth factor receptor during embryogenesis and in adulthood [15]. It is also known that TrkA and NGF were both target genes of KLF7 [4]. These discoveries suggested that KLF7 might participate in neuron regeneration through directly regulating the transcription progress of regeneration-related genes. However, the effects of miR-1b through regulating KLF7 in neuron death-associated diseases still needed further investigation. 
Taken together, the investigations of our study demonstrated that up-regulation of miR-1b could inhibit rat neuron proliferation and regeneration yet promote apoptosis via targeting KLF7. In any event, the involvement of the miRNA system in gene expression regulation for neuron regeneration may be an additional mechanism to fully understand the mechanisms underlying nerve regeneration.

\section{Disclosure}

The authors report no conflict of interest.

\section{References}

1. Azarfar A, Calcini N, Huang C, Zeldenrust F, Celikel T. Neural coding: A single neuron's perspective. Neurosci Biobehav Rev 2018; 94: 238-247.

2. Ben-Yaakov K, Dagan SY, Segal-Ruder Y, Shalem O, Vuppalanchi D, Willis DE, Yudin D, Rishal I, Rother F, Bader M, Blesch A, Pilpel Y, Twiss JL, Fainzilber M. Axonal transcription factors signal retrogradely in lesioned peripheral nerve. EMBO J 2012; 31: 1350-1363.

3. Blackmore MG, Wang Z, Lerch JK, Motti D, Zhang YP, Shields CB, Lee JK, Goldberg JL, Lemmon VP, Bixby JL. Krüppel-like factor 7 engineered for transcriptional activation promotes axon regeneration in the adult corticospinal tract. Proc Nat Acad Sci U S A 2012; 109: 7517-7522.

4. Caiazzo M, Colucci-D’Amato L, Esposito MT, Parisi S, Stifani S, Ramirez F, di Porzio U. Transcription factor KLF7 regulates differentiation of neuroectodermal and mesodermal cell lineages. Exp Cell Res 2010; 316: 2365-2376.

5. Chen X, Liu X, Li B, Zhang Q, Wang J, Zhang W, Luo W, Chen J. Cold Inducible RNA binding protein is involved in chronic hypoxia induced neuron apoptosis by down-regulating HIF-1alpha expression and regulated by microRNA-23a. Int I Biol Sci 2017; 13: $518-531$

6. Ding X, Wang X, Gong Y, Ruan H, Sun Y, Yu Y. KLF7 overexpression in human oral squamous cell carcinoma promotes migration and epithelial-mesenchymal transition. Oncol Lett 2017; 13 2281-2289.

7. Fricker M, Tolkovsky AM, Borutaite V, Coleman M, Brown GC. Neuronal cell death. Physiol Rev 2018; 98: 813-880.

8. Gaudet AD, Mandrekar-Colucci S, Hall JC, Sweet DR, Schmitt PJ, Xu X, Guan Z, Mo X, Guerau-de-Arellano M, Popovich PG. miR155 deletion in mice overcomes neuron-intrinsic and neuron-extrinsic barriers to spinal cord repair. J Neurosci 2016; 36 8516-8532.

9. Hu Z, Li Z. miRNAs in synapse development and synaptic plasticity. Curr Opin Neurobiol 2017; 45: 24-31.

10. Hwang JY, Kaneko N, Noh KM, Pontarelli F, Zukin RS. The gene silencing transcription factor REST represses miR-132 expression in hippocampal neurons destined to die. J Mol Biol 2014; 426: 3454-3466.
11. Indo Y. NGF-dependent neurons and neurobiology of emotions and feelings: Lessons from congenital insensitivity to pain with anhidrosis. Neurosci Biobehav Rev 2018; 87: 1-16.

12. Joly S, Dalkara D, Pernet V. Sphingosine 1-phosphate receptor 1 modulates CNTF-induced axonal growth and neuroprotection in the mouse visual system. Neural Plast 2017; 2017: 6818970.

13. Kajimura D, Dragomir C, Ramirez F, Laub F. Identification of genes regulated by transcription factor KLF7 in differentiating olfactory sensory neurons. Gene 2007; 388: 34-42.

14. Laub F, Lei L, Sumiyoshi H, Kajimura D, Dragomir C, Smaldone S, Puche AC, Petros TJ, Mason C, Parada LF, Ramirez F. Transcription factor KLF7 is important for neuronal morphogenesis in selected regions of the nervous system. Mol Cell Biol 2005; 25: 5699-5711.

15. Lei L, Ma L, Nef S, Thai T, Parada LF. mKIf7, a potential transcriptional regulator of TrkA nerve growth factor receptor expression in sensory and sympathetic neurons. Development 2001; 128: 1147-1158.

16. Li WY, Wang Y, Zhai FG, Sun P, Cheng YX, Deng LX, Wang ZY. AAVKLF7 promotes descending propriospinal neuron axonal plasticity after spinal cord injury. Neural Plast 2017; 2017: 1621629.

17. Li WY, Zhang WT, Cheng YX, Liu YC, Zhai FG, Sun P, Li HT, Deng LX, Zhu XF, Wang Y. Inhibition of KLF7-targeting microRNA 146b promotes sciatic nerve regeneration. Neurosci Bull 2018; 34: 419-437.

18. Li WY, Zhu GY, Yue WJ, Sun GD, Zhu XF, Wang Y. KLF7 overexpression in bone marrow stromal stem cells graft transplantation promotes sciatic nerve regeneration. J Neural Eng 2019; 16: 056011.

19. Li WY, Zhang WT, Cheng YX, Liu YC, Zhai FG, Sun P, Li HT, Deng LX, Zhu XF, Wang Y. Inhibition of KLF7-targeting microRNA 146b promotes sciatic nerve regeneration. Neurosci Bull 2018; 34: 419-437.

20. Liu YP, Xu P, Guo CX, Luo ZR, Zhu J, Mou FF, Cai H, Wang C, Ye XC, Shao SJ, Guo HD. miR-1b overexpression suppressed proliferation and migration of RSC96 and increased cell apoptosis. Neurosci Lett 2018; 687: 137-145.

21. Livak KJ, Schmittgen TD. Analysis of relative gene expression data using real-time quantitative PCR and the 2(-Delta Delta $C(T)$ ) method. Methods 2001; 25: 402-408.

22. Luxenhofer G, Helmbrecht MS, Langhoff J, Giusti SA, Refojo D, Huber AB. MicroRNA-9 promotes the switch from early-born to late-born motor neuron populations by regulating Onecut transcription factor expression. Dev Biol 2014; 386: 358-370.

23. Mahar M, Cavalli V. Intrinsic mechanisms of neuronal axon regeneration. Nat Rev Neurosci 2018; 19: 323-337.

24. McGuire A, Brown JA, Kerin MJ. Metastatic breast cancer: the potential of miRNA for diagnosis and treatment monitoring. Cancer Metastasis Rev 2015; 34: 145-155.

25. Moore DL, Blackmore MG, Hu Y, Kaestner KH, Bixby JL, Lemmon VP, Goldberg JL. KLF family members regulate intrinsic axon regeneration ability. Science 2009; 326: 298-301.

26. Nagata K, Hama I, Kiryu-Seo S, Kiyama H. microRNA-124 is down regulated in nerve-injured motor neurons and it potentially targets mRNAs for KLF6 and STAT3. Neurosci 2014; 256: 426-432.

27. Neumann E, Hermanns H, Barthel F, Werdehausen R, Brandenburger T. Expression changes of microRNA-1 and its targets 
Connexin 43 and brain-derived neurotrophic factor in the peripheral nervous system of chronic neuropathic rats. Mol Pain 2015; 11: 39.

28. Ramkumar A, Jong BY, Ori-McKenney KM. ReMAPping the microtubule landscape: How phosphorylation dictates the activities of microtubule-associated proteins. Dev Dyn 2018; 247: 138-155.

29. Sasi M, Vignoli B, Canossa M, Blum R. Neurobiology of local and intercellular BDNF signaling. Pflugers Archiv 2017; 469: 593-610.

30. van Battum EY, Verhagen MG, Vangoor VR, Fujita Y, Derijck A, O’Duibhir E, Giuliani G, de Gunst T, Adolfs Y, Lelieveld D, Egan D, Schaapveld RQJ, Yamashita T, Pasterkamp RJ. An image-based miRNA screen identifies miRNA-135s as regulators of CNS axon growth and regeneration by targeting Kruppel-like factor 4 J Neurosci 2018; 38: 613-630.

31. Veldman MB, Bemben MA, Thompson RC, Goldman D. Gene expression analysis of zebrafish retinal ganglion cells during optic nerve regeneration identifies KLF6a and KLF7a as important regulators of axon regeneration. Dev Biol 2007; 312: 596 612.

32. Wang Z, Mehra V, Simpson MT, Maunze B, Chakraborty A, Holan L, Eastwood E, Blackmore MG, Venkatesh I. KLF6 and STAT3 co-occupy regulatory DNA and functionally synergize to promote axon growth in CNS neurons. Sci Rep 2018; 8: 12565.

33. Wang Z, Winsor K, Nienhaus C, Hess E, Blackmore MG. Combined chondroitinase and KLF7 expression reduce net retraction of sensory and CST axons from sites of spinal injury. Neurobiol Dis 2017; 99: 24-35.

34. Yi S, Yuan Y, Chen Q, Wang X, Gong L, Liu J, Gu X, Li S. Regulation of Schwann cell proliferation and migration by miR-1 targeting brain-derived neurotrophic factor after peripheral nerve injury. Sci Rep 2016; 6: 29121.

35. Zhou M, Liu X, Li Z, Huang Q, Li F, Li CY. Caspase-3 regulates the migration, invasion and metastasis of colon cancer cells. Int J Cancer 2018; 143: 921-930.

36. Zuo Z, Huang P, Jiang Y, Zhang Y, Zhu M. Acupuncture attenuates renal interstitial fibrosis via the TGFbeta/Smad pathway. Mol Med Rep 2019; 20: 2267-2275. 